\title{
Editorial
}

\section{Data-Driven Fault Supervisory Control Theory and Applications}

\author{
Huaguang Zhang, ${ }^{1}$ Bin Jiang, ${ }^{2}$ and Wen $\mathrm{Yu}^{3}$ \\ ${ }^{1}$ College of Information Science and Engineering, Northeastern University, Shenyang 110004, China \\ ${ }^{2}$ College of Automation Engineering, Nanjing University of Aeronautics \& Astronautics, Nanjing 210016, China \\ ${ }^{3}$ Departamento de Control Automatico, CINVESTAV-IPN, Avenida IPN 2508, 07360 Mexico City, DF, Mexico
}

Correspondence should be addressed to Huaguang Zhang; zhanghuaguang@mail.neu.edu.cn

Received 7 July 2013; Accepted 7 July 2013

Copyright (C) 2013 Huaguang Zhang et al. This is an open access article distributed under the Creative Commons Attribution License, which permits unrestricted use, distribution, and reproduction in any medium, provided the original work is properly cited.

This special issue addresses fault detection, fault diagnosis, and prognosis, which are the three major tasks of data-driven fault supervisory control systems (FSCs). FSCs are desirable in many important real-life applications, from vehicles and aerospace, marine systems, mechatronics, electric power systems, offshore wind technology, and chemical processes to biomedical and other industrial applications involving control. FSCs aim at important goals as to detect potential abnormity, find the causes of abnormity, assess performance degradation tendency, predict the remaining time before a likely failure, and determine failure effects across subsystems.

Fault detection and diagnosis (FDD) has been the subject of intensive research for more than 40 years, and fruitful results have been published in the literature and books. Fault prognosis (FP) is an emerging topic, whose foundation is not yet completely firmed, but whose understanding is getting deeper and deeper. In recent years, data-driven FDD and FP techniques have been attracting intensive attentions both in academic and industrial communities. A number of mature techniques have been given for extracting useful information from measured data. Data-driven FDD and FP techniques have shown their advantages for the complex and large-scale industrial processes and equipments.

The present issue gathers some of the best international specialists and reveals new trend in data-driven FDD, FP, and FSCs. Some significant research problems in FSCs are studied: data-based fault feature extraction, fault reasoning, and decision-making with uncertain information; data-based fault propagation modeling; learning-based fault detection, diagnosis, and supervisory control; hybrid model-based fault accommodation design; new architectures and structures for
FSCs; and applications of FSCs to complex systems. In this special issue, we have received 18 manuscripts, in which 14 manuscripts have been accepted by the reviewers. Four papers are for the problems associated with data-based fault feature extraction, fault reasoning, and decision-making; five papers are on the topic of learning-based fault detection, diagnosis, and supervisory control; two papers are for the fault propagation/prediction of complex systems, and three papers are for the fault diagnosis of nonlinear complex systems.

Four papers in this special issue studied the problems associated with data-based fault feature extraction, fault reasoning, and decision-making with uncertain information. In the paper by J. He and C. F. Zhang, the authors used the coordinate transformation algorithm to decouple the system to eliminate the influence of disturbances to the system for better disturbance robustness. With the equivalent principle of sliding mode variable structure, the reconstruction of arbitrary nonlinear faults was achieved to isolate and estimate faults. In the work conducted by J. Chen et al., a quantitative assessment approach to COTS (commercial off-the-shelf) component security is proposed. The steps of interface fault injection and the assessment framework were given based on the internal factors of the tested component. The quantitative assessment algorithm and formula of component security level were also presented. G. Wang et al. developed a fuzzy hyperbolic with time-varying delays that guaranteed cost controller design via state-feedback for a class of nonlinear continuous-time systems with parameter uncertainties. Meanwhile, the authors presented some sufficient conditions for the existence of such a fuzzy hyperbolic by a set of linear matrix inequalities (LMIs). In the work of M. He et al., a new 
fault detection method based on froth texture unit distribution (TUD) was proposed to recognize the fault condition of sulfur flotation in real time. In order to overcome the difficulties of the traditional kernel basis that cannot compare different TUD under various conditions, a nonparametric kernel estimator was designed to approximate the TUD curve, which is the probability density function (PDF) of froth texture unit number. By transforming the nonparametric description into dynamic kernel weight vectors, a principle component analysis (PCA) model based on those dynamic kernel weight vectors was established to realize the performance recognition.

Five papers worked on the topic of learning-based fault detection, diagnosis, and supervisory control. X.-L. Xia et al. proposed an algorithm combining the Least-Squares Support Vector Machine (LS-SVM) and the Forward Least-Squares Approximation (FLSA) algorithm. By adopting FLSA and reformulating the classical LS-SVM, a sparse regression model was obtained. The proposed algorithm is verified on a number of datasets, and the results showed that the proposed algorithm outperformed the conventional SVMs. Y. Zhang et al. presented a new modeling approach for process monitoring in the electrofused magnesia furnace (EFMF), where a new technique for extracting the common subspace of different modes in EFMF was proposed based on a manifold learning method. The extracted common subspace was shared by all different modes. After two different subspaces were separated, the common and specific subspace models were built and analyzed, respectively. In the paper by J. Liu et al., a new adaptive fuzzy min-max (AFMN) neural network classifier was proposed. Combined with principle component analysis (PCA) and adaptive genetic algorithm (AGA), the integrated system can realize supervised and real-time classification. S. Thenozhi et al. studied the health monitoring systems for tall buildings in order to evaluate possible damages. The authors used two techniques for structural health monitoring of tall buildings: numerical integrator and convexconcave hull classification. The authors used Jarvis march method to decide the concave (nonconvex) hull for the inseparable points. In the work by $\mathrm{H}$. Gong and Z. Zhen, a new nonlinear NN-augmented observer was presented to approximate the unmodeled dynamics instead of the entire system. The proposed neural observer can generate residuals that are essential for fast fault detection.

Two papers are for the fault propagation/prediction of complex systems. N. Lu et al. considered the issue of databased fault propagation/prediction. The authors first proposed a technique for specifying the causality and estimating the time delays among process variables. Such information is critical for finding the root cause of a plant-wide disturbance and revealing the evolution of an abnormal event propagating through the plant. Then a fault prognosis strategy was presented on the basis of time-delayed digraph (TD-SDG) model and PCA, where the TD-SDG model is used to represent both information directions and information delays among process variables and PCA is used to realize the earlier fault detection. Their method was verified by applying to an air separation unit for predicting the frequently occurred "nitroen block" fault. G. Yangming et al. aimed at time series online prediction of complex system and proposed a new method to improve the prediction accuracy based on grey system theory and incremental learning algorithm.

The remaining three papers are for the fault diagnosis of nonlinear complex systems. The paper by A. Cuervo et al. developed a new structure for FSCs, in order to solve the limitations that the existing FDI schemes for nonlinear stochastic dynamical systems cannot behave correctly with presences of both additive and parametric faults. A new integrated scheme for fault detection and isolation was then developed for dealing with simultaneous additive and parametric faults. Simulation results were presented to confirm the theoretical studies. The paper written by Z. Wang et al. designed a state estimation observer and a fault diagnosis observer for a class of discrete-time delayed complex interconnected networks with linear coupling, which can estimate the system state and detect the actuator fault, respectively. The state observer was based on the linear matrix inequality method, while the fault diagnosis observer used an adaptive strategy. The paper by X. $\mathrm{Li}$ and H. H. T. Liu considered the smallest gain of system sensitivity in fault detection. A necessary and sufficient condition on the lower bound of $\mathrm{H}$-index infinite time horizon for linear discrete time-varying systems is developed. It is characterized in terms of the existence of solution to a backward difference Riccati equation with an inequality constraint. In addition, a similar condition in terms of algebraic Riccati equation in frequency domain is developed for linear discrete time-invariant systems in infinite time horizon. In comparison with the wellknown bounded real lemma, it is found that $\mathrm{H}$ index is not completely dual to $\mathrm{H} 1$ norm.

We hope that this special issue will offer a useful reference for people working in fault detection, diagnosis, and prognosis areas and help academics, scientists, and engineers explore new methodologies in both theory investigation and practical applications.

\section{Acknowledgments}

We would like to thank all the contributors and reviewers for their excellent work and timely support, which make the special issue possible and successful. In particular, we are greatly grateful to the editor of this journal to give us the opportunity to edit this special issue and all the very helpful advice during the process.

Huaguang Zhang Bin Jiang Wen $\mathrm{Yu}$ 


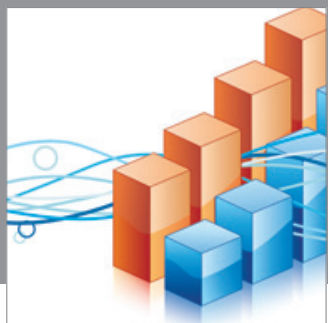

Advances in

Operations Research

mansans

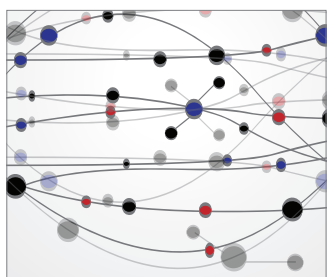

The Scientific World Journal
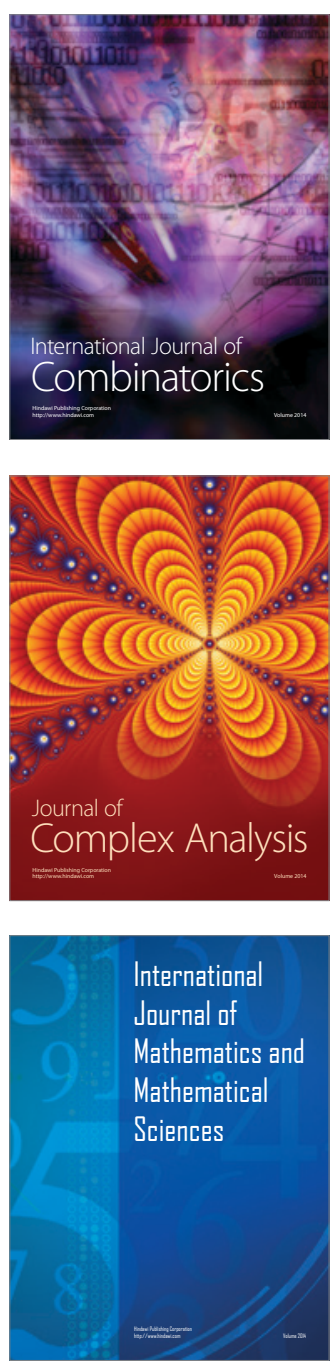
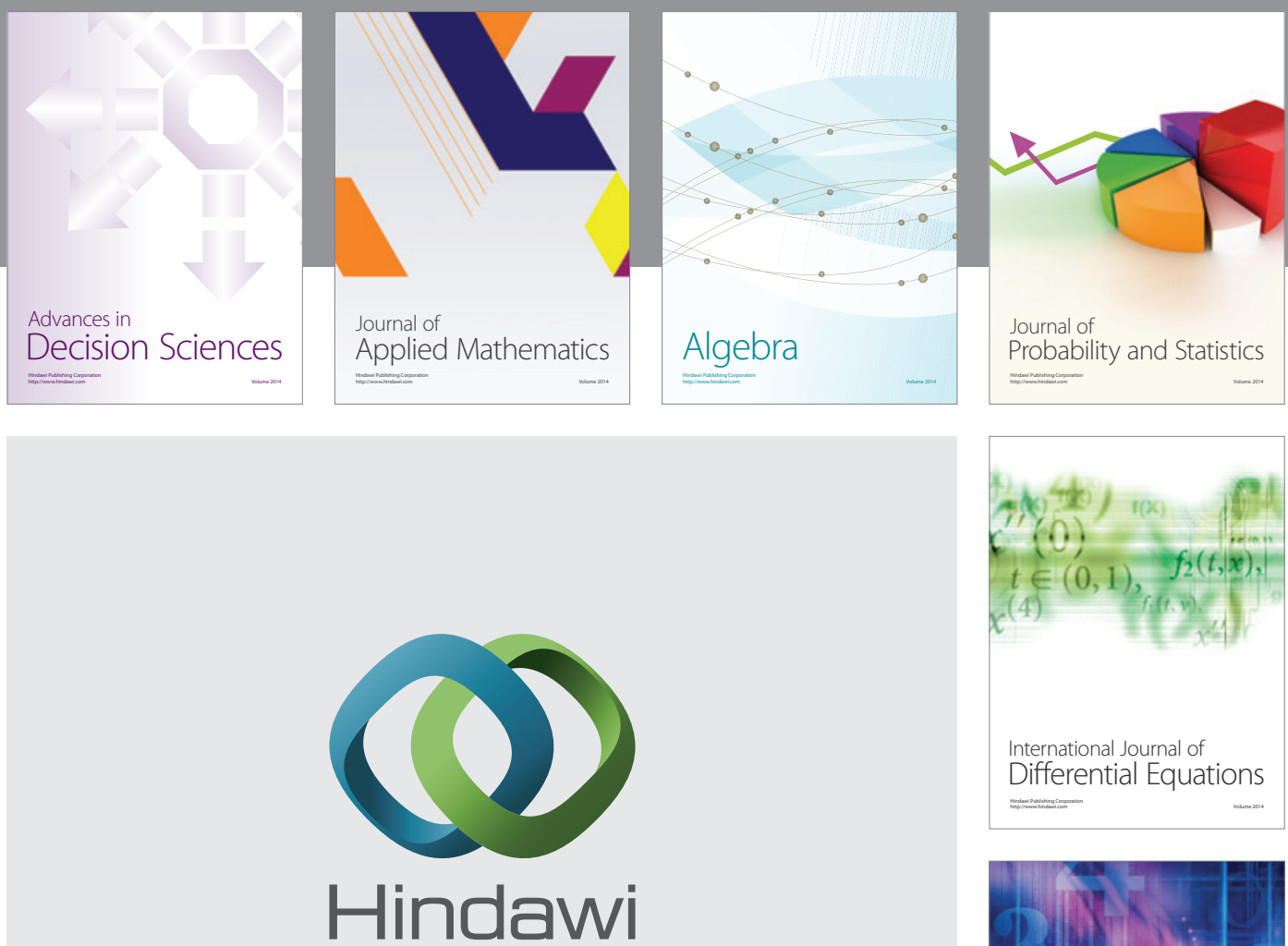

Submit your manuscripts at http://www.hindawi.com
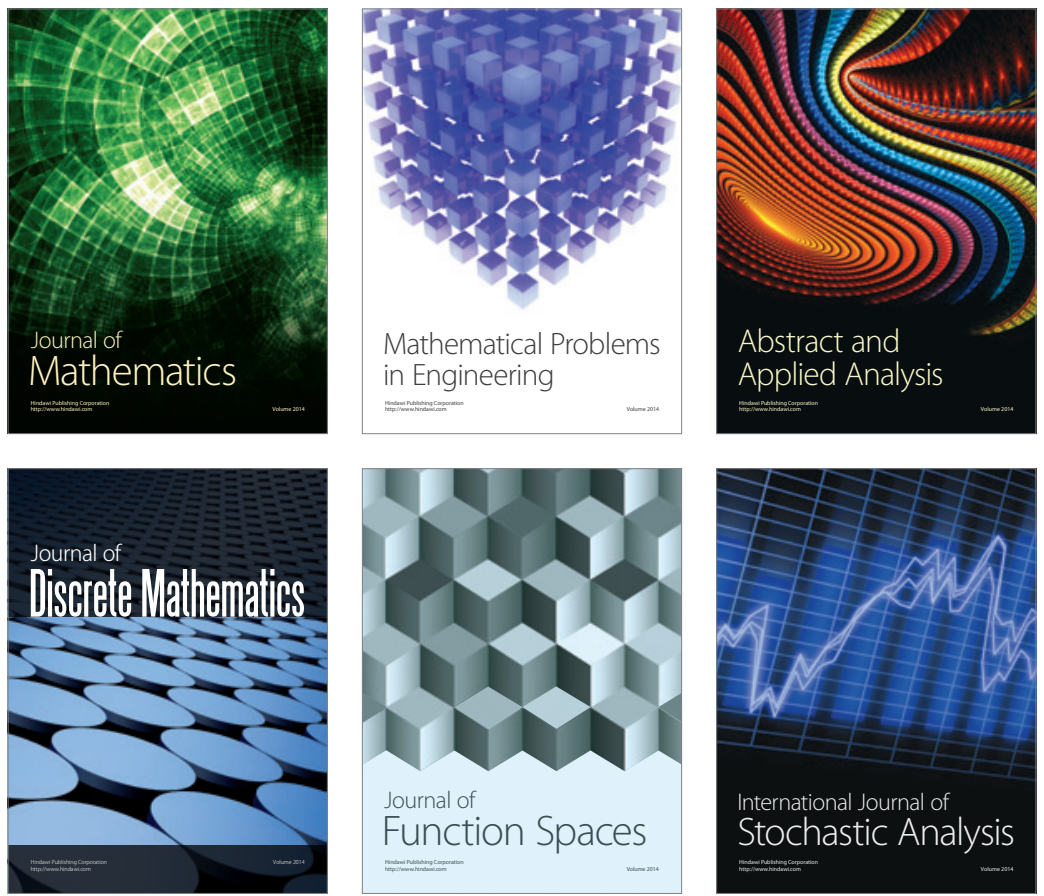

Journal of

Function Spaces

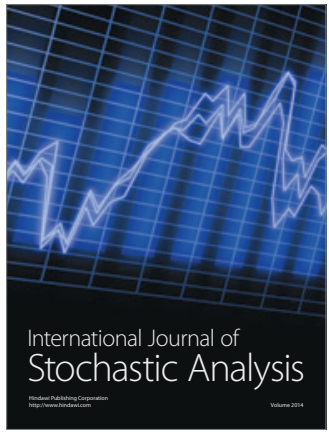

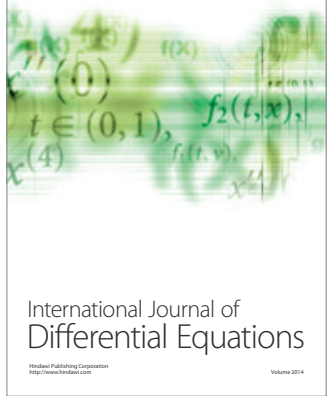
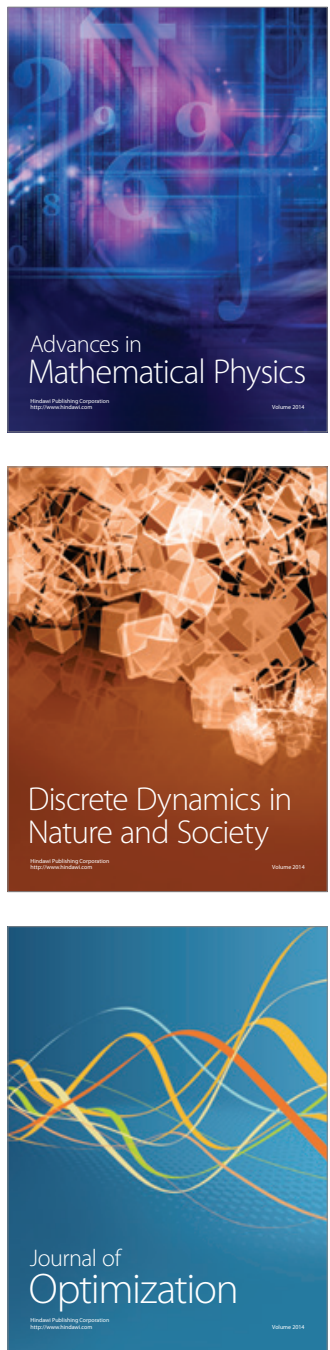